\title{
Optimal Design of a Dragonfly-Inspired Compliant Joint for Camera Positioning System of Nanoindentation Tester Based on a Hybrid Integration of Jaya-ANFIS
}

\author{
Ngoc Le Chau, ${ }^{1}$ Thanh-Phong Dao $\mathbb{D}^{2,3}$ and Van Thanh Tien Nguyen ${ }^{1}$ \\ ${ }^{1}$ Faculty of Mechanical Engineering, Industrial University of Ho Chi Minh City, Ho Chi Minh City, Vietnam \\ ${ }^{2}$ Division of Computational Mechatronics, Institute for Computational Science, Ton Duc Thang University, Ho Chi Minh City, Vietnam \\ ${ }^{3}$ Faculty of Electrical \& Electronics Engineering, Ton Duc Thang University, Ho Chi Minh City, Vietnam \\ Correspondence should be addressed to Thanh-Phong Dao; daothanhphong@tdt.edu.vn
}

Received 7 March 2018; Accepted 9 July 2018; Published 26 July 2018

Academic Editor: Aimé Lay-Ekuakille

Copyright (C) 2018 Ngoc Le Chau et al. This is an open access article distributed under the Creative Commons Attribution License, which permits unrestricted use, distribution, and reproduction in any medium, provided the original work is properly cited.

\begin{abstract}
Camera positioning system is a critical member of a nanoindentation tester characterizing the mechanical properties such as hardness, creep, surface roughness, or elastic modulus of a material sample. This paper presents a design optimization for a dragonfly-inspired compliant joint. This joint is used to drive the camera positioning system. A new hybrid approach of Taguchi method, adaptive neuro-fuzzy inference system (ANFIS), and Jaya algorithm is developed to solve the multi-objective optimization problem. The Taguchi method is used to build the numerical data and to find the best membership functions for the ANFIS structure by minimizing the root mean squared error. Then, the weight factor of each objective function is determined by established equations well. Subsequently, a structure of ANFIS is developed to map the design parameters and responses. Sensitivity analysis of each controllable parameter is analyzed by the statistical method. Finally, Jaya algorithm is initialized to find the optimal solution. The results found that the optimal displacement, frequency, and stress are about $12581.11 \mu \mathrm{m}, 67.76 \mathrm{~Hz}$, and $333.68 \mathrm{MPa}$, respectively. The proposed hybrid optimization algorithm is a robust and effective optimizer and considered as soft computing technique for engineering optimization problems.
\end{abstract}

\section{Introduction}

In the recent decades, the nanoindentation tester has been widely used in areas of academic and industrial investigations. The practitioners and researchers used this technique to probe the mechanical properties of hard and soft materials such as hardness, creep, elastic-plastic modulus, roughness surface, etc.

During the indentation process, the camera is used to record the image of material sample before and after indenting test. The camera often requires a high positioning precision and a stability to figure out the curve of displacement versus load. In commercialization, the current instruments are effective but so costly. The reasons are that the screw and servomotors are commonly used to ensure the characterization precision. Besides, a force and displacement sensor are separately utilized to record the load and indentation depth.
It has been facing a difficult challenge for researchers to make cheaper system but better accuracy.

To eliminate the cost and enhance the positioning accuracy, a new dragonfly compliant joint is proposed to drive the camera positioning system. The proposed joint has no backlash, free friction, high precision, and monolithic structure [1-3]. Inversely, the servo-driven system has the backlash and friction between kinematic joints resulting in increasing the cost and reducing the precision. Another advantage of the joint is that it can be integrated with strain gauges to directly measure the force, displacement, or stress. It is therefore to eliminate the commercial sensors.

For the real nanoindentation tester [4], a camera positioning system does not require a large displacement but also needs a faster response. According to the elastic theory, the new joint operates in the elastic area without failures. As a result, a resulting stress within the joint should ensure a 
long working strength. Recently, the microscopes also are potential cameras to replace to allow an automatic imaging process and decrease focus positioning error and operation's influences. It can find various applications such as cell microscopy [4], imaging [5], scanning microscopy metrology and nanolithography $[6,7]$, cellular camera phone [8], the image sharpness identification [9], laser probe system [10], and imaging cameras [11-13]. However, these systems have still some limitations due to their heavy weight and complex design.

Mimicking animal's function of motion, animal-inspired mechanisms were developed to gain a light weight and a better compliance. For example, a few studies focused on jumping robots [14], prosthetic hand [15], bio-inspired structural materials [16], leg design for running [17], compliant spine for climbing [18], avian-inspired rotorcraft [19], quadrotor perching [20], bio-mechatronic robot hand [21], bio-inspired condylar hinge joint for mobile robots [22], six-legged robot [23], flea-inspired jumping mechanism [24], vibration energy harvest [25], precision robots [26], bio-inspired wing flappers [27], bio-inspired soft wearable robot [28], and legged insect-scale robot [29], and, in the old version, the authors introduced a compliant thin-walled joint based on zygoptera's profile [30]. Inspired by the human muscle motions, camera positioning devices allowed a fast-moving robot eye. For instance, several were driven by traditional servo motors with rigid links [31-33]. This principle made a fast motion but used servomotors with heavy weights that could make a significant discrepancy. In order to suppress these limitations, a new dragon-inspired compliant joint is recommended in the present work for the camera positioning system. Up to now, there have not been the studies on the dragonfly compliant joint as the driver for camera positioning system in nanoindentation tester. The joint is designed based on the demands of the system. The performances are then improved by developing the hybrid optimization method.

The new contribution of this study is to develop a new hybrid optimization approach of Taguchi method, adaptive neuro-fuzzy inference system (ANFIS), and Jaya. The proposed method is used to optimize the design parameters of the proposed joint. To meet the requirements of nanoindentation tester, the camera positioning system should permit a large working travel, a high speed, and a good strength. Of course, these objectives conflicted each other, and the proposed optimization approach is therefore developed as an efficient decision-maker to trade-off among these objectives.

In general, Taguchi method was a robust optimization technique but only used effectively for single objective [3134]. For general optimization problems, mathematical models are established, and then an evolutionary optimization algorithm is utilized to find the best solution. However, if the established equations are a wrong approximation, the optimized results are not corrected. For the dragonfly compliant joint, it includes so many linear and nonlinear parameters in mathematical models. This is hence difficult to establish the mathematical models. As a result, a black-box is required to accurately approximate the relation between design variables and responses. Therefore, the ANFIS is adopted to map well the inputs and outputs based on human knowledge in the form of fuzzy if-then rules and stipulated input-output data. The Jaya is then developed to optimize the multiple objective problem. In this study, a weight factor is calculated accurately to assign the priority to each response. A logically systematic approach of this work is considered as a soft computing technique facilitating the academy to the industry.

The goal of this paper is to propose an effective optimization algorithm for the dragonfly compliant joint. The experimental data is collected firstly. The Taguchi method is used to identify the best membership functions for the ANFIS by minimizing the root mean squared error. And then, ANFIS's structure is developed to approximate the pseudomathematical models. The weight factor is then determined well by statistical-based equations. Subsequently, the Jaya is used to for optimization process. Finite element analysis and experimental validations are conducted to verify the effectiveness and robustness of the hybrid optimization.

\section{Design of Camera Positioning System for Nanoindentation Tester}

2.1. Design of Dragonfly Compliant Joint. For a real nanoindentation tester, camera positioning system (CPS) is assigned to detect and inspect the observed material sample before and after indentation process. The signal is then transferred to a measurement system to recognize the mechanical properties of the sample. Nowadays, a screw is integrated with a servo motor to achieve a high positioning accuracy for the CPS. However, it often requires strictly manufacturing and assembling and this causes a large cost.

To eliminate the cost and increase the production process, a new dragonfly compliant joint (DCJ) was recommended as an alternative candidate for the screw and servomotor. The DCJ also transferred force or displacement but its positioning precision was higher and the cost of manufacture was relatively cheaper. The reasons were that the DCJ is monolithically manufactured, free friction and lubricant, and reduced assembling. Besides, the DCJ would displace accurately in a few micrometers to hundreds of micrometers.

Unlike the group of traditional joints, the DCJ would permit a large deformation to store and release a better strain energy. Also, it can be said that a successful CPS begins with a designing well for the DCJ. Practically, the system does not only need a large workspace so as to inspect a large indentation depth but also requires a high resonant frequency in order to increase the speed of the system. Therefore, this paper concentrated on the optimal design for the DCJ.

Figures 1(a)-1(c) depict the dragonfly animal, its wing, and the proposed DCJ, respectively. This ideal was replied on the wing's profile of the animal. From the wing's curve, a lot of points of $\mathrm{p}_{\mathrm{i}}(\mathrm{i}=1,2,3, \ldots, \mathrm{n})$ were created even and they were connected in series to become a nonlinear lower wing of the DCJ. And then, the existing curve was mirrored in a symmetric topology to form the upper wing. Subsequently, other wings would be made similarly to enlarge the length of the joint. Noted that there was a free space at the middle structure of the DCJ, and it made a thickness $t$ for the joint. The DCJ can allow becoming more flexible in degrees of 
TABLE 1: Parameters of the DCJ.

\begin{tabular}{|c|c|c|c|c|c|}
\hline Properties of $\mathrm{Al} 7075$ & Value & Unit & Geometric dimensions & Value & Unit \\
\hline Young's modulus & 71000 & $\mathrm{MPa}$ & $p$ & 22.92 & $\mathrm{~mm}$ \\
\hline Yield strength & 503 & $\mathrm{MPa}$ & $l$ & 57. 45 & $\mathrm{~mm}$ \\
\hline Density & 2770 & $\mathrm{Kg} / \mathrm{m}^{3}$ & $W$ & 38 & $\mathrm{~mm}$ \\
\hline \multirow[t]{5}{*}{ Poisson ratio } & 0.3 & & $e$ & 15 & $\mathrm{~mm}$ \\
\hline & & & $t$ & Variable & $\mathrm{mm}$ \\
\hline & & & $d_{1}$ & Variable & $\mathrm{mm}$ \\
\hline & & & $d_{3}$ & Variable & $\mathrm{mm}$ \\
\hline & & & $d_{4}$ & Variable & $\mathrm{mm}$ \\
\hline
\end{tabular}

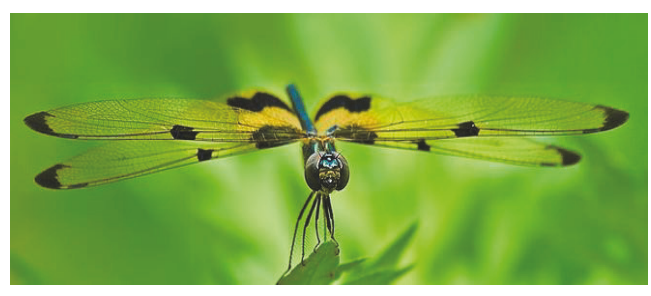

(a)

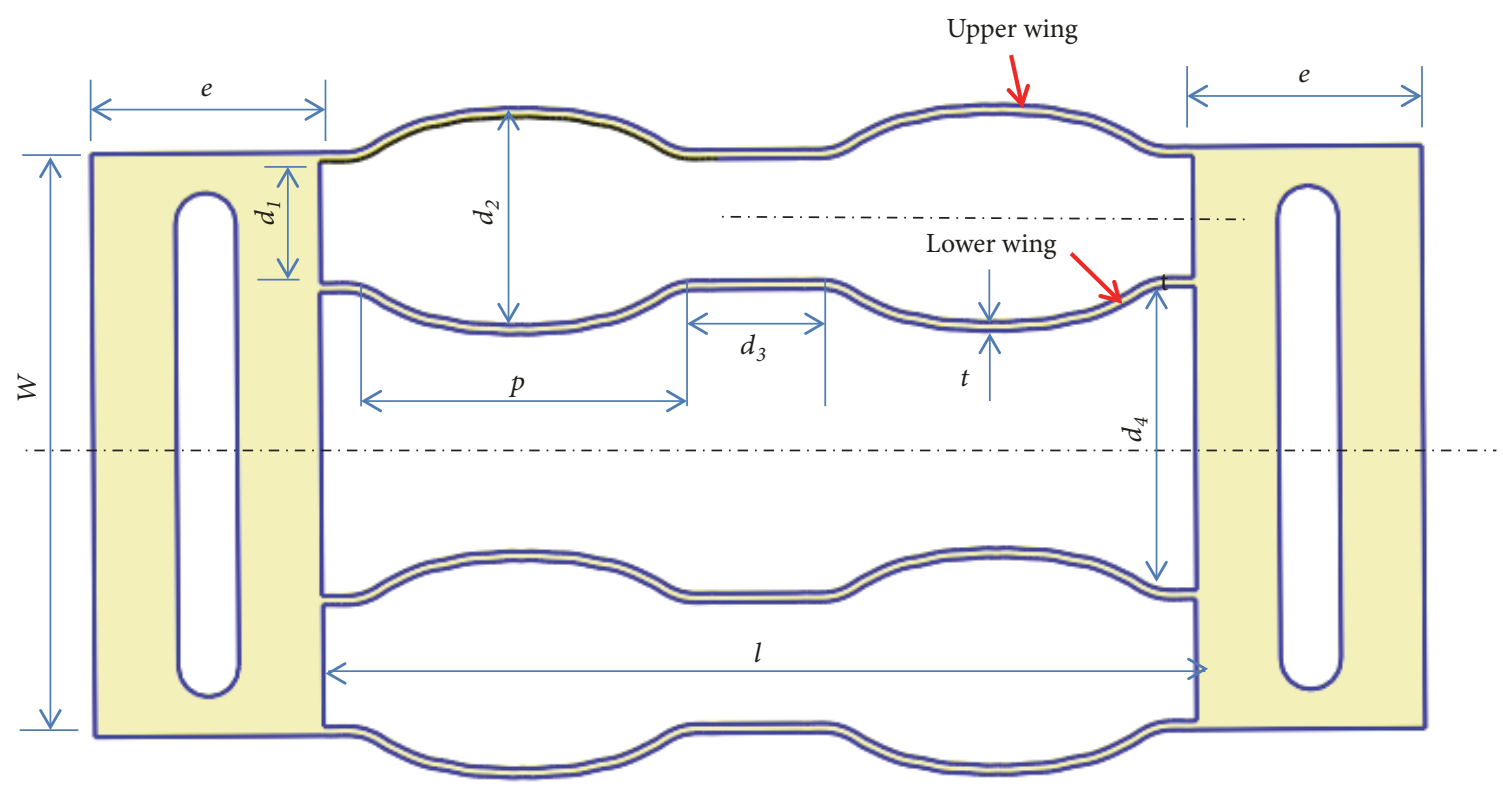

(c)

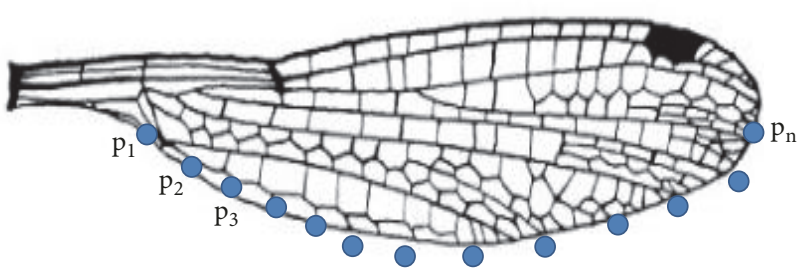

(b) 


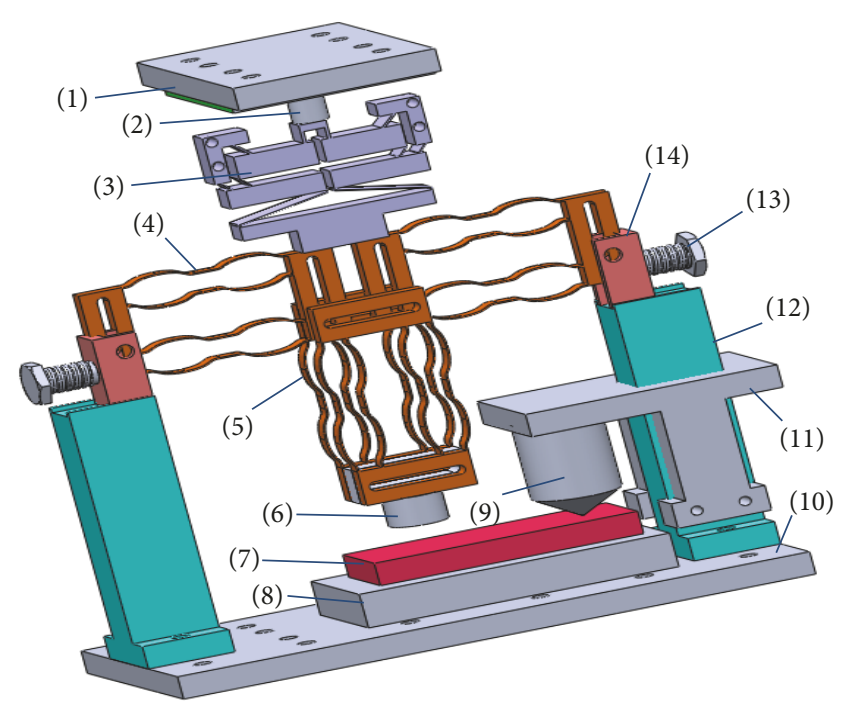

FIGURE 2: Assemble model for nanoindentation testing system.

During the indentation process, overall system should be suppressed any inner-and-outer vibrations so that the imaging quality would be stable, accurate, and more clear. To solve this problem, a vibration isolator was integrated with the CPS. The principle of the isolator was based on a quasizero stiffness. This type includes two vertical springs with positive stiffness are coupled with two horizontal springs with negative stiffness to form overall stiffness of the system which was almost zero. From that, it could harvest vibrations and make a stable CPS for nanoindentation testing system.

Figure 2 illustrates a potential application of the CPS for nanoindentation tester. It includes 14 parts with different functions. The top plate (1) was fixed by screws. An actuator (2) like voice coil motor was placed on the top plate (1) and it generated a force to a multiple lever-based amplification mechanism (3) so as to amplify the working travel of the CPS.

Two DCJs (4) were considered as the horizontal spring and had a negative stiffness $k_{n}$. Besides, two DCJs (5) were assigned as the vertical spring (5) with a positive stiffness $k_{p}$. The total of vibration isolator was approximately $K_{s}$ $=k_{p}-k_{n}=0$. It helped to initialize a quasi-zero stiffness vibration isolator. A camera (6) was fixed at the end of DCJs (5). The camera tends to test the material sample (7) prior to indentation process. And then, the high precision $\mathrm{XY}$ positioning stage (8) would bring the sample moving the position of the indenter (9). The indenter was driven using the accurate precision $\mathrm{Z}$ positioning stage. The CPS, $\mathrm{XY}$ stage, and $\mathrm{Z}$ stage were displaced in the range from a few micrometers to hundreds of micrometers. The overall system of nanoindentation tester is supported by two supporters (12) and bottom plat (10). Two screws (13) were used to adjust and remain overall weights and system at equilibrium station replied on two sliders (14).

To improve the performances of the joint, a new hybrid optimization method was proposed later. This approach was based on the Taguchi robust parameter design, the ANFIS,

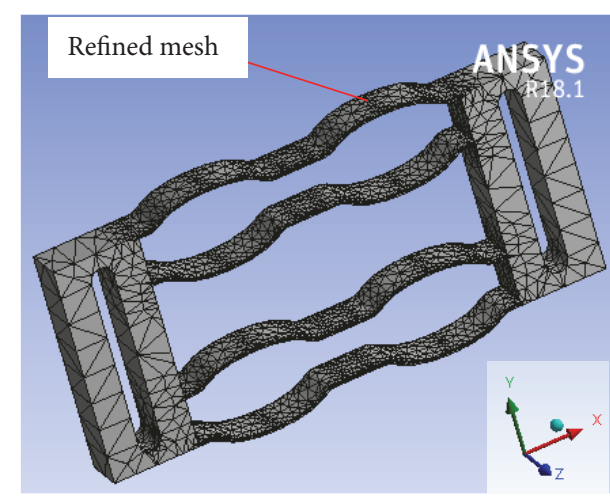

FIgURE 3: Meshed model for the DCJ.

and the Jaya, which was considered as a soft computing technique.

\section{Simulations and Experiments}

Several facing challenges of DCJ are herein how to allow a large displacement to enlarge an indentation depth, a high frequency to increase the speed, and a long working time. Therefore, this study concentrated on designing, analyzing, and optimizing these characterizations. First of all, the data were extracted from simulations and experiments.

A finite element analysis (FEA) was implemented in the software ANSYS 18.1 to retract the simulation results. The mesh model of DCJ was refined to allow an accurate result, as seen in Figure 3. It was noted that the resulting stress of DCJ should be lower than the yield strength of Al 7075. Hence, this study retrieved the stress via simulations and experiments. According to the elastic mechanics, the stress is computed based on the strain deformation.

During the current nanoindentation system, the force and displacement signal are recorded separately by sensors. This causes a waste in cost and human sources and instruments. To eliminate the limitations, the DCJ can be integrated with strain gauges so as to measure the displacement, force, strain, and stress by its self.

The suitable locations for attaching the strain gauges on the surfaces of the DCJ would be firstly determined. Assume that the eight positions of DCJ were very sensitive to the strain gauges, which included $\mathrm{S}_{1}, \mathrm{~S}_{2}, \mathrm{~S}_{3}, \mathrm{~S}_{4}, \mathrm{~S}_{5}, \mathrm{~S}_{6}, \mathrm{~S}_{7}$, and $\mathrm{S}_{8}$, as shown in Figure 4 . Also, the boundary conditions, including the fixed support and external force $F$, were given in Figure 4. One end of the DCJ was fixed and free end was moved along the y-axis by the load $F$. The simulation results indicated that the maximum strains appeared at the upper surfaces of DCJ such as $S_{1}, S_{7}, S_{2}$, and $S_{8}$, on the wing's curve, as seen in Figure 5.

In order to build the sensor, a half-Wheatstone bridge circuit was used for the strain gauge, as shown in Figure 6. The elastic theory and this circuit were combined to develop the mathematical equation for measuring the actual stress. As seen in Figure 6, if the DCJ was under tension, the resistance $R$ would increase by a value of $\Delta R$. Conversely, in the case of 


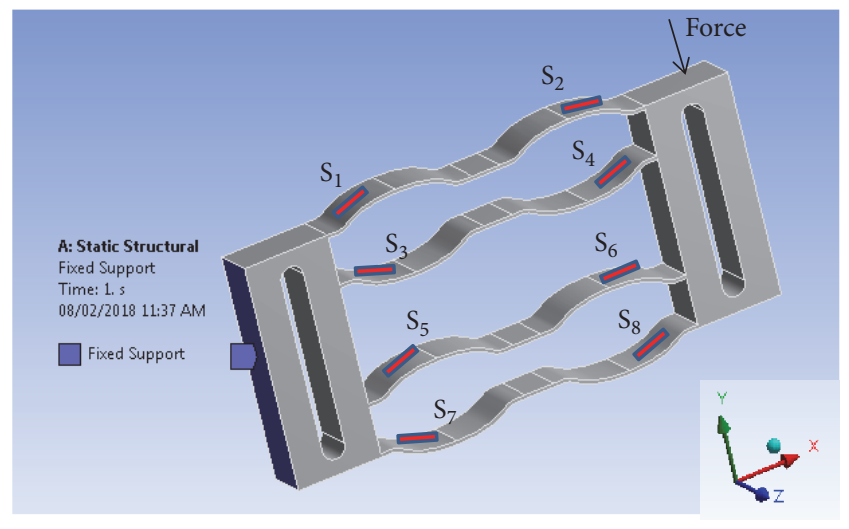

Strain gauge

Figure 4: Possible positions of strain gauge and boundary conditions.

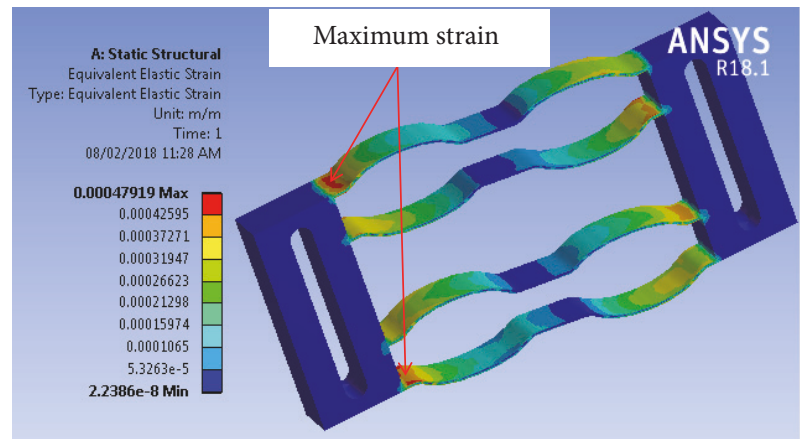

FIGURE 5: Distribution of the strain.

compression, the resistance $R$ would decrease by a value of $\Delta R$.

The gauge factor of the strain gauge was determined as the ratio of fractional change in the electrical resistance to the fractional change in length:

$$
G=\frac{\Delta R}{R \times \varepsilon},
$$

where $G$ represents the gauge factor of the strain gauge, $\Delta R$ indicates the change value of the gauge resistance, $R$ is the nominal value of the gauge strain, and $\varepsilon$ is the real strain.

The actual stress was computed by the following equation:

$$
\sigma=E \times \varepsilon,
$$

where $\sigma$ is the stress and $E$ is Young's modulus of the material $\mathrm{Al} 7075$.

Finally, the experiment for measuring the strain was performed, as depicted in Figure 7. The strain gauges were glued on the surface of $S_{1}$ and $S_{7}$. A force gauge was used to apply a force to the free end of DCJ. And then, data from the sensor gauges were transferred into the DAQ instrument, which generated data into the CPU. LABVIEW ${ }^{\circledR}$ software was set up in the CPU so that the strain wave and value of the strain were displayed on the screen. The strain value after



FIGURE 6: Half-Wheatstone bridge circuit of the strain gauge.

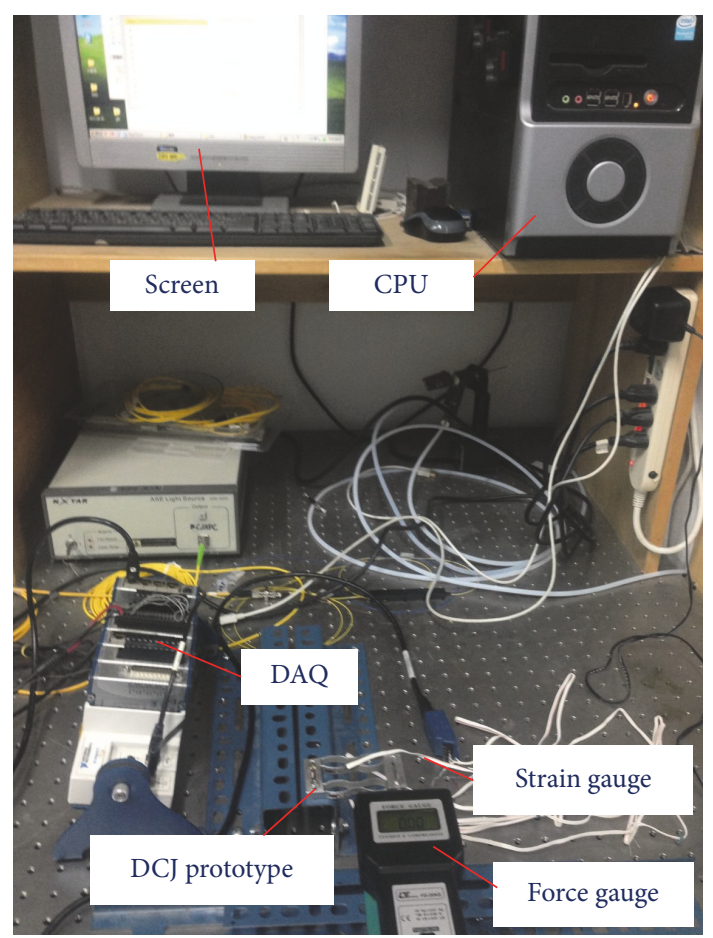

FIGURE 7: Real Strain measurement of the DCJ.

each experimental measure was recorded. The actual strain was repeated five times and the average value was calculated. The actual stress value was calculated using (2).

Considering determination of the displacement of the DCJ, Figure 8 depicts the experimental measurement of the displacement of DCJ in the y-axis. A force gauge was used to adjust up to $10 \mathrm{~N}$. A laser displacement sensor (KEYENCE, Japan) was then utilized to retrieve the displacement. A connector was utilized to transfer the signal to a digital display. 




FIGURE 8: Displacement of DCJ in y-axis.

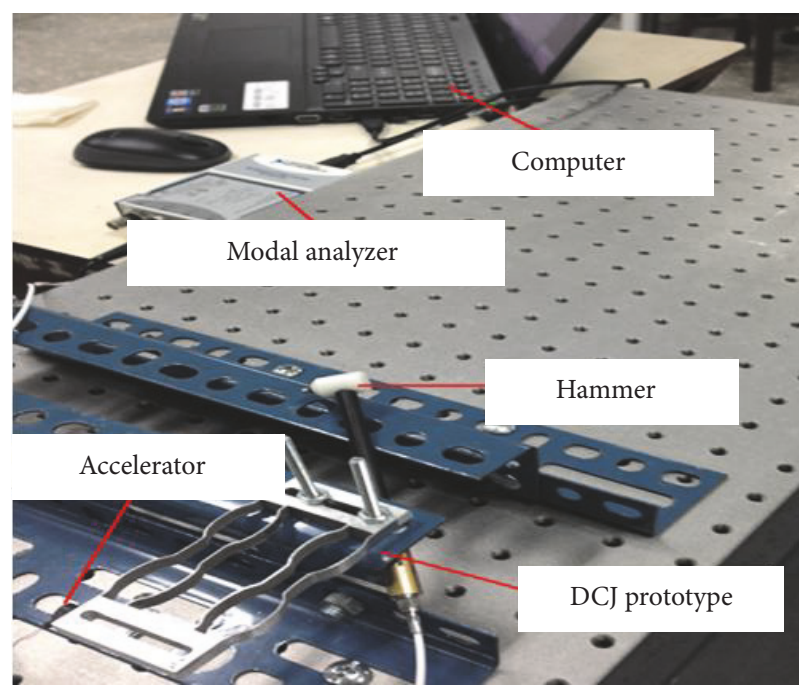

FIGURE 9: Frequency measurement of DCJ.

Regarding frequency measurement for the DCJ, a modal hammer (Model 9722A2000-SN 2116555) from KISTLER was used to apply the excitation, and the frequency response was measured by using an accelerator (Model 4744892) from KISTLER. Noted that the accelerator should be attached to be opposite the excitation from the modal hammer, as given in Figure 9.

\section{Formulation of Optimization Problem}

Current nanoindentation tester system is suitable for various materials such as biological tissues and soft gels. The question covered herein whether the commercialized instruments can monitor easily the mechanical properties of a sample but its operating costs are rather high. In some cases where a large indentation depth is required, the existing cameras are difficult to fulfill higher precise probe requirements in several tens of micrometers to hundreds of millimeters. Hence, the considerable challenges have been faced when developing the nanoindentation devices; the instruments should have the following specifications:

(i) Being able to measure a large indentation depth

(ii) Speeding up the indentation process

(iii) Ensuring a long working time

(iv) Gaining an excellent stability

(v) Being able to record the displacement and force signal itself before and after indentation process.

In this study, a new nanoindentation testing system was designed in which the basic part, the CPS, was proposed to improve five aforementioned specifications above. Specifically, the CPS could gain a large displacement, a high speed, and a long working time. It was noted that the proposed DCJ was very sensitive to geometric dimensions such as lengths $d_{1}, d_{3}$, and $d_{4}$ and thickness $t$. Hence, these design variables should be optimized to improve the displacement, frequency, and stress of DCJ. In this system, the DCJ should fulfill the following important requirements to satisfy facing challenges as follows:

(a) A high first natural frequency to permit a faster speed and to avoid a resonance with an excited frequency of actuators

(b) A large working stroke to allow enough displacement of the CPS

(c) A small resulting stress to achieve a good strength criterion.

In general, these requirements were conflicted together. The question that was raised here is how to trade-off multiple responses, simultaneously. As a result, a new hybrid optimization approach was developed to solve the multi-objective problem later.

4.1. Design Variables. The vector of design variables was considered as $\mathbf{X}=\left[t, d_{1}, d_{3}, d_{4}\right]^{T}$. The lower and upper bounds for the design variables were assigned according to designer's experiences and capacity of wire discharged machining process, which were described as follows:

$$
\begin{gathered}
0.4 \mathrm{~mm} \leq t \leq 0.8 \mathrm{~mm} \\
5 \mathrm{~mm} \leq d_{1} \leq 11 \mathrm{~mm} \\
4 \mathrm{~mm} \leq d_{3} \leq 10 \mathrm{~mm} \\
14 \mathrm{~mm} \leq d_{4} \leq 20 \mathrm{~mm},
\end{gathered}
$$

where $t$ and $d_{i}$ with $i=1,3,4$ represent the thickness and length, respectively.

4.2. Objective Functions. Retrieving from real system, the following multiple objective functions were considered: (i) for the first objective function, $f_{1}(\mathbf{X})$, the displacement is desired as large as possible; (ii) for the second objective function, $f_{2}(\mathbf{X})$, a high first natural frequency is to increase the speed; and (iii) for third objective function, $f_{3}(\mathbf{X})$, a 
minimum stress was required. In summary, the optimization problem was described as follows.

Design variables were considered as $\mathbf{X}=\left[t, d_{1}, d_{3}, d_{4}\right]^{T}$.

$$
\begin{aligned}
& \max f_{1}(\mathbf{X}) \\
& \max f_{2}(\mathbf{X}) \\
& \min f_{3}(\mathbf{X})
\end{aligned}
$$

As was aforementioned, these objective functions would be transferred into a scalar single objective function to search an optimal Pareto set. Then, all objective functions were normalized and made the combined objective function. At this stage, the normalized combined objective function was stated as follows:

$$
f(\mathbf{X})=-w_{1} \times f_{1}(\mathbf{X})-w_{2} \times f_{2}(\mathbf{X})+w_{3} \times f_{3}(\mathbf{X}),
$$

where $w_{1}, w_{2}, w_{3}$ are the normalized weight factors of displacement, frequency, and stress, respectively. Signal "-" in (7) means that the corresponding objective functions were maximized. Signal " + " shows a minimized value.

For any multiple objective optimization problem, the quality response is very sensitive to the weight factor. As a result, the weight factor would be calculated accurately in later.

4.3. Constraints. The DCJ would work in an elastic limit of the Al-7075; it was subjected to constraints as follows:

$$
g(x)=\sigma \leq \frac{\sigma_{y}}{S},
$$

where $\sigma_{y}$ is the yield strength of material; $S$ is the safety factor of $S=1.5$ as selected in order to guarantee the long working life.

\section{Hybrid Optimization Algorithm}

As was aforementioned, the conflict cost functions could be treated as a Pareto optimal solution. Therefore, a new hybrid approach was developed to resolve the multiple criteria optimal design for the proposed DCJ. First, the experimental data was collected. Then, the ANFIS architecture was developed to map inputs and outputs. Finally, Jaya algorithm was dedicated to optimizing multiple objective responses, simultaneously. The programming was executed in MATLAB R2015b. A flowchart for optimizing the DCJ was described in Figure 10. The important thing was how to achieve a robust solution and easy to implement.

Phase 1 (collect the data). The experimental data would consist of both the displacement and frequency. The number of experiments was assigned via using an orthogonal array of the Taguchi method. Then, Taguchi method is also applied to identify the best membership functions for the structure ANFIS in this study by optimizing the root mean squared error.

Phase 2 (determine the weight factor). As discussed above, the displacement, first natural frequency, and gripping effort

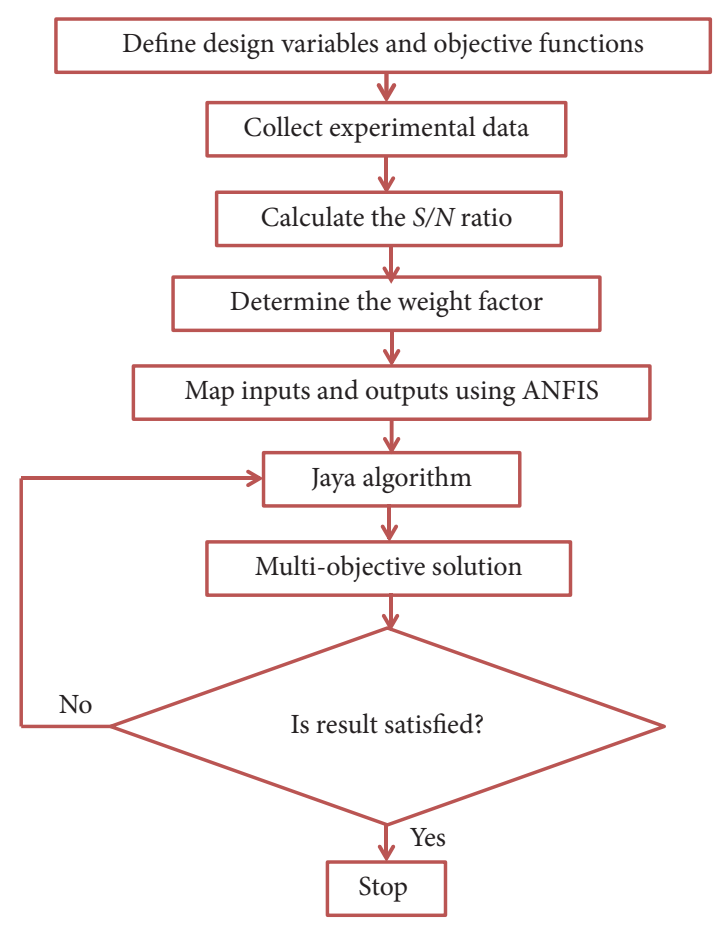

FIGURE 10: Flowchart of multi-objective optimization procedure for the DCJ.

were conflicted. Moreover, they had different important weights. Each objective function should put a weight factor (WF). Up to now, to solve a Pareto optimal solution, the WF was determined based on a designer' experiences and institution. This would lead to a wrong optimized result because the WF had a significant importance related directly to a decision-maker.

To solve the WF accurately, this paper proposed an approach based on the value of $S / N$ ratios. One of the most important steps is that each objective function should be normalized in the range $[0,1]$, which can be considered as $z_{i}\left(0 \leq z_{i} \leq 1\right)$. The WF can be calculated step by step as follows.

Step 1 (calculate signal-to-noise ratio). The TM deals with the signal-to-noise $(S / N)$ ratio that shows a better performance corresponding to a larger $S / N$ ratio value. The Taguchi robust parameter design categories depend on the desired performance response. In this paper, there were three objective functions that were considered for designing the DCJ, as follows: (i) a large displacement was desired, (ii) a high first natural frequency was expected, and (iii) a minimum stress was required.

The larger-the better was used for the displacement and frequency, which was defined as follows:

$$
\eta=-10 \log \left(\frac{1}{n} \sum_{i=1}^{n} \frac{1}{y_{i}^{2}}\right)
$$


The smaller-the better was utilized for the stress as

$$
\eta=-10 \log \left(\frac{1}{n} \sum_{i=1}^{n} y_{i}^{2}\right),
$$

where $y$ is the quality characteristics; $n$ the number of replicates of experiment ith.

Step 2 (normalize the $S / N$ ratio). The $S / N$ ratios of each level of each objective function can be normalized as follows:

$$
z_{i}=\frac{\eta_{j}-\min \eta_{j}}{\max \eta_{j}-\min \eta_{j}},
$$

where $z_{i}$ is the normalized value of $S / N$ for the experiment ith of the objective function $(j=1,2, \ldots, k), k$ is the number of objective functions, $\eta_{i}$ indicates the $S / N$ value, and $\max \eta_{i}$ and $\min \eta_{i}$ are the largest and smallest values of $\eta_{i}$, respectively.

Step 3 (calculate the average value of normalized $S / N$ ratios (AVONSNR)). The AVONSNR for each objective function was calculated. After normalizing the $S / N$ ratio, the AVONSNR can be determined by following equation:

$$
a_{L i}=\frac{1}{N_{L i}} \sum_{i}^{m} z_{i j},
$$

where $a_{L i}$ is the average value of $S / N$ ratio of level ith of each design variable of each objective function, $N_{L j i}$ is the number of repetitions of level ith, and $\eta_{i j}$ is the value of $S / N$ ratio of level ith of objective function $\mathrm{jth}$.

Step 4 (compute the range of each design variable). The range (max-min) of the each level of each design variable was determined as follows:

$$
\begin{aligned}
r_{i j}= & \max \left\{z_{i, j, 1}, z_{i, j, 2}, \ldots, z_{i, j, m}\right\} \\
& -\min \left\{z_{i, j, 1}, z_{i, j, 2}, \ldots, z_{i, j, m}\right\},
\end{aligned}
$$

where $r_{i j}$ is the range of the $S / N$ ratio for each level of each parameter, $j=1,2, . . p, p$ is the number of design variables, and $m=1,2, . . l, l$ is the number of experimental levels of each design variable.

Step 5 (determine the WF). The WF was calculated by the following:

$$
w_{i}=\frac{\sum_{j=1}^{p} r_{i j}}{\sum_{i=1}^{m_{\mathrm{OF}}} \sum_{j=1}^{p} r_{i j}},
$$

where $w_{i}$ is the WF of each objective function ith and $w_{i} \geq$ 0 . $m_{\mathrm{OF}}$ is the number of objective functions.

In general, sum of WF for overall objective functions is equal to one as follows:

$$
\sum_{i=1}^{m_{\mathrm{OF}}} w_{i}=1
$$

Phase 3 (adaptive neuro-fuzzy inference system). Sooner, fuzzy logic is an inexact reasoning enabling modeling of the human reasoning process in linguistic terms [35]. In this theory, the Sugeno fuzzy model has been widely used to create fuzzy rules if there are an input and output data. And then, a basic ANFIS was proposed [36]. ANFIS model was one of the implementations of a first-order Sugeno fuzzy inference system. The rules were formed as follows:

$$
\begin{aligned}
& \text { if } x_{1} \text { is } A_{1} \text { and } x_{2} \text { is } A_{2} \text {, } \\
& \text { then } y=p \times x_{1}+q \times x_{2}+r
\end{aligned}
$$

where $x_{1}$ and $x_{2}$ are inputs corresponding to $A_{1}$ and $A_{2}$ term set, $y$ is output, and others parameters $p, q$, and $r$ are constant.

A structure of ANFIS includes five-layer feed-forward neural network as follows:

(1) Layer 1 was considered as the fuzzification layer that carried out a fuzzification for the inputs to decide the membership degrees for each input based on the given fuzzy membership function. Outputs of this layer can be identified as follows:

$$
M_{1}^{i}=\mu A_{i}(x)
$$

where $x$ is input to node $i$, and $A_{i}$ is linguistic label associated with this node function. $M_{1}^{i}$ is the membership function of $A_{\mathrm{i}}$.

(2) Layer 2 was the rule, a rule node, collecting inputs from the respective fuzzification nodes and determining the firing strength of the rule. Each node in this layer is a circle node named $П$. Each node output represents the firing strength of a rule. It can be described as follows:

$$
w_{i}=\mu A_{i}(x) \times \mu A_{i}(y) \ldots \quad i=1,2,3, \ldots, N
$$

(3) Layer 3 was defined as the normalized layer evaluating the ratio of the firing strength of a given rule to the total of firing strengths of all rules. Every node in this layer is a circle node symbolized $N$. The $i$ th node determines the ratio of the $i$ th rules firing strength to the sum of all rule's firing strengths, where $\bar{w}$ is the normalized firing strength of rules.

$$
\bar{w}=\frac{w_{i}}{w_{1}+w_{2}+w_{3}, \ldots,+w_{n}}, \quad i=1,2,3, \ldots, N
$$

(4) Layer 4 was the defuzzification layer receiving initial inputs and giving the consequent parameters of the rule. Every node $i$ in this layer is a square node as follows:

$M_{1}^{i}=\bar{w}_{i} f_{i}(p x+q y, \ldots, r), \quad i=1,2,3, \ldots, N$

where $\bar{w}_{i}$ is the output of layer 3 and $p, q, r$ are the parameter set. 


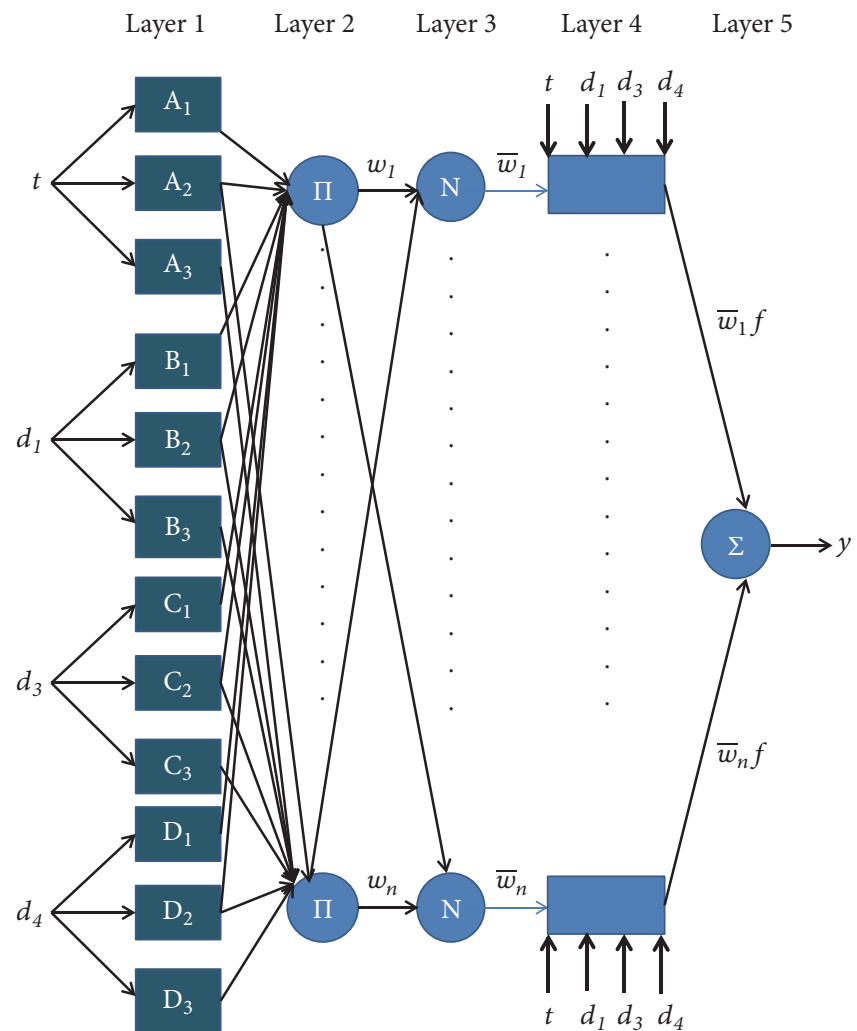

Adaptive

Fixed node

FIGURE 11: Proposed ANFIS for the DCJ.

(5) Layer 5 was a single node calculating the overall output as the sum of all incoming signals, as follows:

$$
M_{5}^{i}=\sum_{i} \bar{w}_{i} f_{i} \frac{\sum_{i} w_{i} f_{i}}{w_{i}}
$$

In the ANFIS training, the least squares were commonly combined with a gradient descent algorithm. In the algorithm, each epoch on the hybrid procedure composed a forward pass and a backward pass. In a forward pass, a training set of inputs was almost suggested to the ANFIS and neuron outputs were determined by the layer-by layer basis. The consequent parameters were computed by the least squares algorithm. The ANFIS structure often utilizes the typical membership functions such as Gaussian, sigmoidal, triangular, trapezoidal, bell types, and so on. At least a difficulty was how to find the most suitable membership functions. In this paper, the structure of ANFIS for the DCJ was developed, as given in Figure 11.

As shown in Figure 11, five network layers were used by ANFIS to perform the following fuzzy inference steps: (1) input fuzzification, (2) fuzzy set database construction, (3) fuzzy rule base construction, (4) decision-making, and (5) output defuzzification.
Phase 4 (Jaya algorithm). Jaya algorithm was proved as a more effective approach due to its convergent speed when compared with metaheuristic optimization algorithms. Therefore, the Jaya [37] was used in this paper to optimize both the displacement and frequency for the CMG. The Jaya procedure is illustrated, as in Figure 12.

The Jaya mimics the behavior of biological species to survive in its environments. In the Jaya, the most successful member was considered as the best solution. Meanwhile, the unsuccessful member was understood as the worst solution. In this approach, initial solutions were retrieved randomly according to in the range of design parameters. And then, each design variable of every solution was updated by the following equation:

$$
\begin{aligned}
U_{a+1, b, c}= & U_{a, b, c}+\gamma_{a, b, 1}\left(U_{a, b, b e s t}-a b s\left(U_{a, b, b e s t}\right)\right) \\
& -\gamma_{a, b, 2}\left(U_{a, b, w o r s t}-a b s\left(U_{a, b, w o r s t}\right)\right),
\end{aligned}
$$

where $a, b, c$ represent the index of iteration, design variable, and candidate solution. $U$ shows the $b$ th design variable of $c$ th candidate in $a$ th iteration. Moreover, $\gamma, a, b, 1$ and $\gamma, a, b$, 2 mean the generated randomly numbers in the range $[0,1]$, which were regarded as scaling factors. 


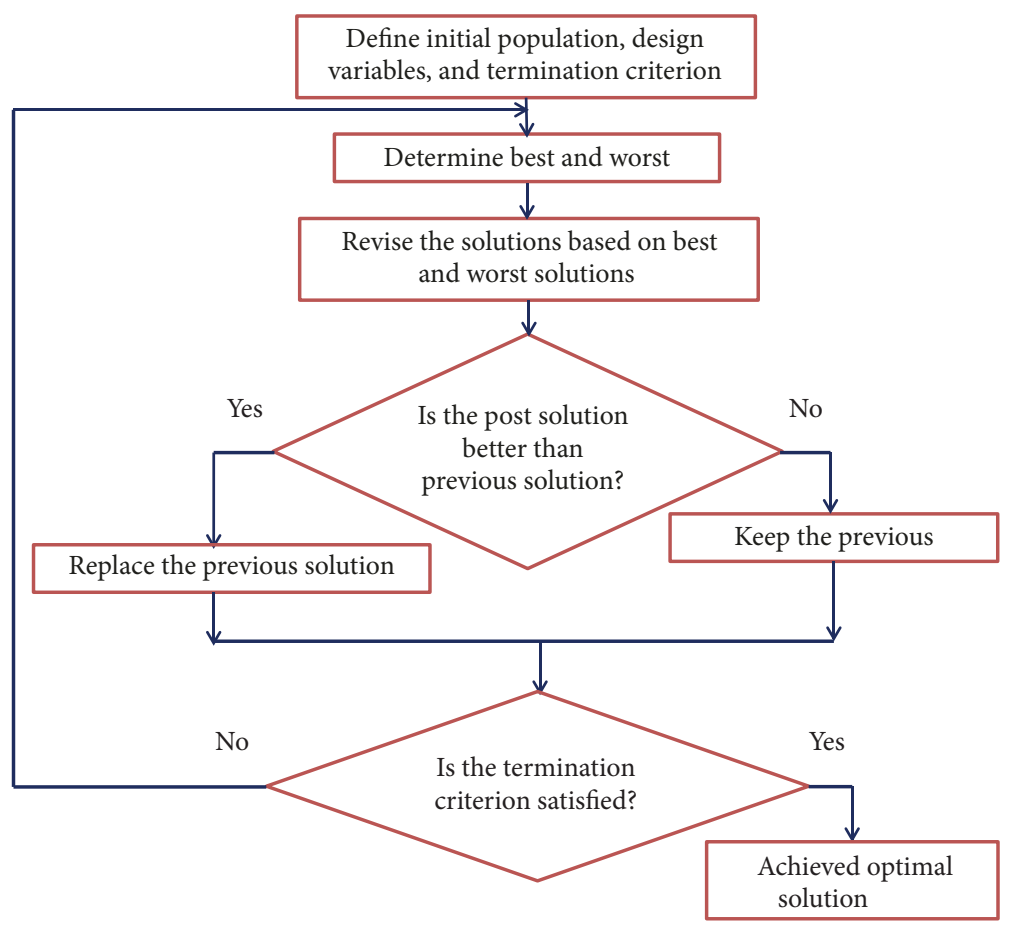

Figure 12: Procedure of the Jaya.

TABle 2: Parameters and their levels.

\begin{tabular}{lccccc}
\hline \multirow{2}{*}{ Factor } & \multirow{2}{*}{ Symbol } & Unit & \multicolumn{3}{c}{ Levels } \\
& & & 1 & 2 & 3 \\
\hline$t$ & $\mathrm{~A}$ & $\mathrm{~mm}$ & 0.4 & 0.6 & 0.8 \\
$d_{1}$ & $\mathrm{~B}$ & $\mathrm{~mm}$ & 5 & 8 & 11 \\
$d_{3}$ & $\mathrm{C}$ & $\mathrm{mm}$ & 4 & 7 & 10 \\
$d_{4}$ & $\mathrm{D}$ & $\mathrm{mm}$ & 14 & 17 & 20 \\
\hline
\end{tabular}

\section{Results and Discussion}

The eight design variables were divided into three levels, as given in Table 2 . The experimental data was collected using $L_{9}$ orthogonal array, as shown in Table 3.

To determine the best membership functions (MFs) for the ANFIS structure of DCJ, various MFs were compared based on the root mean square error (RMSE) by the following equation:

$$
R M S E=\sqrt{\frac{1}{m} \sum_{i=1}^{m}\left(t_{i}-y_{i}\right)^{2}}
$$

where $m$ is the number of observations. $t_{i}$ and $y_{i}$ denote the actual and predicting value, respectively.

The Taguchi method was used herein to determine the best MFs for the ANFIS structure in this study by minimizing the RMSE from (23). The experimental data from Table 3 were used for training and five random set of data were utilized for testing of ANFIS. The results showed that the $\pi$-shaped MFs is the best for ANFIS model of DCJ because its RMSE is smallest, as given in Table 4. As a result, it was selected for this study.

The mathematical model of the $\pi$-shaped MFs is formed as follows:

$$
f(x, a, b, c, d) \begin{cases}0, & x \leq a \\ 2\left(\frac{x-a}{b-a}\right)^{2}, & a \leq x \leq \frac{a+b}{2} \\ 1-2\left(\frac{x-b}{b-a}\right)^{2}, & \frac{a+b}{2} \leq x \leq b \\ 1, & b \leq x \leq c \\ 1-2\left(\frac{x-c}{d-c}\right)^{2}, & c \leq x \leq \frac{c+d}{2} \\ 2\left(\frac{x-d}{d-c}\right)^{2}, & \frac{c+d}{2} \leq x \leq d \\ 0, & x \geq d\end{cases}
$$

where parameters $a$ and $d$ locate the feet of the curve, while $b$ and $c$ locate its shoulders.

The resulting parameters of ANFIS for DCJ were found in Table 5. These parameters are actually difficult to determine by establishing the mathematical models. Hence, the relationship between design variables and three responses can be identified via using ANFIS. If a mathematical model is formed, the results may be not correct. There were so many parameters that cannot define accurately if a regression approach was used. Summarily, ANFIS was the best approach for the proposed DCJ.

The experimental data were then transferred to the $S / N$ ratios using (9) and (10), as shown in Table 6. Next, the $S / N$ ratios were normalized using (11), as given in Table 7. 
TABLE 3: Experimental results.

\begin{tabular}{|c|c|c|c|c|c|c|c|}
\hline No. & $\begin{array}{c}t \\
(\mathrm{~mm})\end{array}$ & $\begin{array}{c}d_{1} \\
(\mathrm{~mm})\end{array}$ & $\begin{array}{c}d_{3} \\
(\mathrm{~mm})\end{array}$ & $\begin{array}{c}d_{4} \\
(\mathrm{~mm})\end{array}$ & $\begin{array}{c}\text { Displacement } \\
f_{1}(\mu \mathrm{m})\end{array}$ & $\begin{array}{c}\text { Natural frequency } \\
f_{2}(\mathrm{~Hz})\end{array}$ & $\begin{array}{c}\text { Stress } \\
f_{3}(\mathrm{MPa}) \\
\end{array}$ \\
\hline 1 & 0.4 & 5 & 4 & 14 & 10287.0 & 73.893 & 314.19 \\
\hline 2 & 0.4 & 8 & 7 & 17 & 11241.0 & 62.314 & 307.99 \\
\hline 3 & 0.4 & 11 & 10 & 20 & 3120.4 & 76.949 & 137.62 \\
\hline 4 & 0.6 & 5 & 7 & 20 & 1810.9 & 111.72 & 186.4 \\
\hline 5 & 0.6 & 8 & 10 & 14 & 4003.1 & 109.76 & 149.01 \\
\hline 6 & 0.6 & 11 & 4 & 17 & 2125.0 & 117.95 & 156.39 \\
\hline 7 & 0.8 & 5 & 10 & 17 & 916.48 & 153.37 & 109.41 \\
\hline 8 & 0.8 & 8 & 4 & 20 & 688.35 & 161.96 & 94.595 \\
\hline 9 & 0.8 & 11 & 7 & 14 & 798.14 & 137.73 & 100.41 \\
\hline
\end{tabular}

TABLE 4: Comparison of RMSE for various membership functions for the joint.

\begin{tabular}{lcc}
\hline Membership functions & & RMSE \\
\hline Triangular MFs (trimf) & Training data & Testing data \\
Trapezoidal MFs (trapmf) & 0.030347 & 0.030347 \\
Generalized bell MFs (gbellmf) & 0.030242 & 0.030242 \\
Gaussian MFs (gaussmf) & 0.035087 & 0.035087 \\
Gaussian combination MFs (gauss2mf) & 0.031246 & 0.031246 \\
$\pi$-shaped MFs (pimf) & 0.030244 & 0.030244 \\
Difference between two sigmoidal MFs (dsigmf) & 0.029883 & 0.029883 \\
Product of two sigmoidal MFs (psigmf) & 0.03039 & 0.03039 \\
\hline
\end{tabular}

TABLE 5: ANFIS results for the joint.

\begin{tabular}{lc}
\hline ANFIS results & Number of elements \\
\hline Number of nodes & 551 \\
Number of linear parameters & 256 \\
Number of nonlinear parameters & 48 \\
Total number of parameters & 304 \\
Number of training data pairs & 9 \\
Number of testing data pairs & 5 \\
Number of fuzzy rules & 256 \\
\hline
\end{tabular}

TABLE 6: The values of $S / N$ ratios.

\begin{tabular}{lccc}
\hline No. & $\begin{array}{c}S / N \text { of } \\
\text { displacement }(\mathrm{dB})\end{array}$ & $\begin{array}{c}S / N \text { of Frequency } \\
(\mathrm{dB})\end{array}$ & $S / N$ of stress $(\mathrm{dB})$ \\
\hline 1 & 80.2458 & 37.3721 & -49.9438 \\
2 & 81.0161 & 35.8917 & -49.7707 \\
3 & 69.8842 & 37.7241 & -42.7736 \\
4 & 65.1579 & 40.9626 & -45.4089 \\
5 & 72.0479 & 40.8089 & -43.4643 \\
6 & 66.5472 & 41.4340 & -43.8842 \\
7 & 59.2425 & 43.7148 & -40.7811 \\
8 & 56.7562 & 44.1882 & -39.5174 \\
9 & 58.0416 & 42.7806 & -40.0355 \\
\hline
\end{tabular}

In this table, the normalized $S / N$ ratios for displacement $\left(\eta_{1}\right)$, frequency $\left(\eta_{2}\right)$, and stress $\left(\eta_{3}\right)$ were $Z_{1}, Z_{2}$, and $Z_{3}$,
TABle 7: The normalized $S / N$ ratios $\left(z_{i}\right)$.

\begin{tabular}{lccc}
\hline No. & $Z_{1}$ of $\eta_{1}$ & $Z_{2}$ of $\eta_{2}$ & $Z_{3}$ of $\eta_{3}$ \\
\hline 1 & 0.9682 & 0.1784 & 0.0000 \\
2 & 1.0000 & 0.0000 & 0.0020 \\
3 & 0.5411 & 0.2209 & 0.0835 \\
4 & 0.3463 & 0.6112 & 0.0528 \\
5 & 0.6303 & 0.5927 & 0.0755 \\
6 & 0.4036 & 0.6680 & 0.0706 \\
7 & 0.1025 & 0.9429 & 0.1067 \\
8 & 0.0000 & 1.0000 & 0.1215 \\
9 & 0.0530 & 0.8303 & 0.1154 \\
\hline
\end{tabular}

respectively. Using (12)-(14), the WF for each response was computed, as given in Tables 8,9 , and 10 , respectively. The WF of displacement was about 0.4895 , the WF of the frequency was 0.4504 , and the WF of the stress of 0.0601 . Sum of WFs was equal to one. In general, the WF for each response was assigned 0.5 but this value was not correct. This would lead to a wrong optimized solution. Therefore, this paper proposed a new approach to determine the accurate the value of WFs. Also, it revealed that the displacement has the most important degree, followed by the frequency and smallest stress.

After achieving the WFs and the relation of design variables and three cost functions were formed, the JA was used to optimize the DCJ. The optimal program was carried out using Matlab. The parameters of JA were initialized as population size of 30 and tolerance of $10^{-6}$. The optimal 


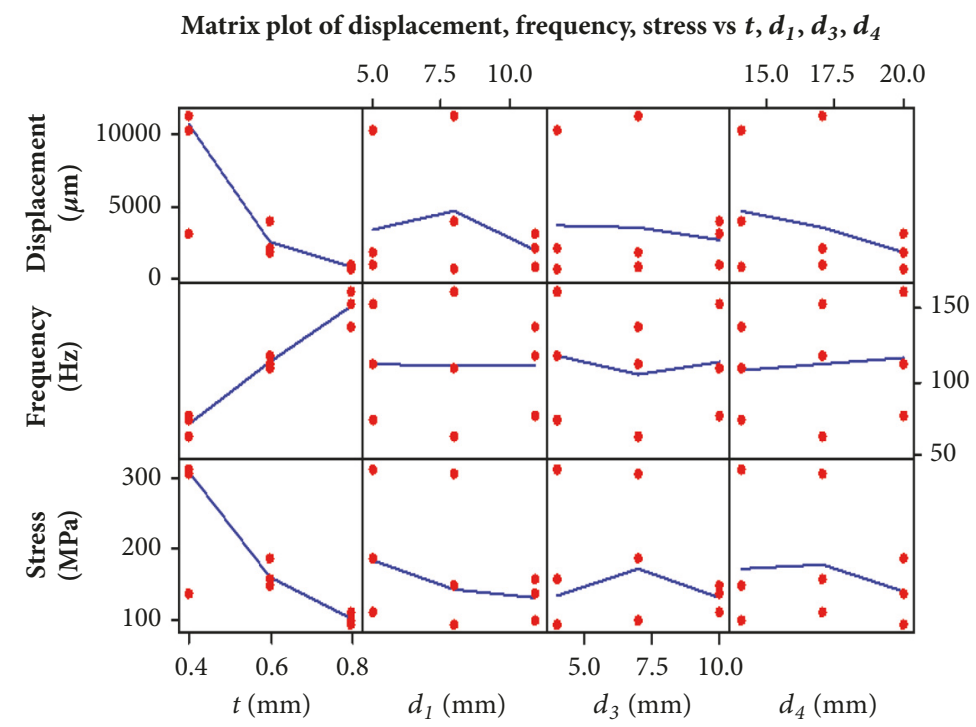

FIGURE 13: Sensitivity diagram of the controllable parameters on the responses.

TABLE 8: The weight factor for the displacement.

\begin{tabular}{lcccc}
\hline \multirow{2}{*}{ Level } & \multicolumn{5}{c}{ The mean value of normalized $S / N$ ratios of each } \\
& $\mathrm{A}$ & $\mathrm{B}$ & $\mathrm{C}$ & $\mathrm{D}$ \\
\hline Level 1 & 0.8365 & 0.1575 & 0.1524 & 0.1835 \\
Level 2 & 0.4601 & 0.1811 & 0.1555 & 0.1673 \\
Level 3 & 0.0518 & 0.1109 & 0.1416 & 0.0986 \\
Range $r_{i j}$ & 0.7846 & 0.0703 & 0.0139 & 0.0849
\end{tabular}

Weight factor for the displacement: $w_{1}=0.4895$

TABLE 9: The weight factor for the frequency.

\begin{tabular}{lcccc}
\hline \multirow{2}{*}{ Level } & \multicolumn{5}{c}{ The mean value of normalized $S / N$ ratios of each } \\
& $\mathrm{A}$ & $\mathrm{B}$ & $\mathrm{C}$ & $\mathrm{D}$ \\
\hline Level 1 & 0.1331 & 0.1925 & 0.2052 & 0.1779 \\
Level 2 & 0.6240 & 0.1770 & 0.1602 & 0.1790 \\
Level 3 & 0.9244 & 0.1910 & 0.1952 & 0.2036 \\
Range $r_{i j}$ & 0.7913 & 0.0155 & 0.0450 & 0.0256
\end{tabular}

Weight factor for the frequency: $w_{2}=\mathbf{0 . 4 5 0 4}$

results were generated at the generation of 44 . The optimal design variables were found at $\mathrm{x}_{\mathrm{val}}=\left[t=0.4, d_{1}=6.96, d_{3}\right.$ $\left.=6.91, d_{4}=15.64\right]$, as seen in Table 11. Also, the results found that the optimal displacement, frequency, and stress are about $12581.11 \mu \mathrm{m}, 67.76 \mathrm{~Hz}$, and $333.68 \mathrm{MPa}$, respectively. These values were better than those of using the teaching learningbased optimization algorithm (TLBO) in the comparison. Hence, the Jaya-based ANFIS was an efficient technique. The optimal performances satisfied the real requirements and therefore the optimal process was ended herein.
TABLE 10: The weight factor for the stress.

\begin{tabular}{lcccc}
\hline \multirow{5}{*}{ Level } & \multicolumn{5}{c}{ The mean value of normalized $S / N$ ratios of each } \\
& $\mathrm{A}$ & $\mathrm{B}$ & $\mathrm{C}$ & $\mathrm{D}$ \\
\hline Level 1 & 0.0285 & 0.0177 & 0.0213 & 0.0212 \\
Level 2 & 0.0663 & 0.0221 & 0.0189 & 0.0199 \\
Level 3 & 0.1146 & 0.0295 & 0.0295 & 0.0286 \\
Range $r_{i j}$ & 0.0860 & 0.0118 & 0.0106 & 0.0087
\end{tabular}

Weight factor for the stress: $w_{3}=0.0601$

TABLE 11: Comparison of emerging optimization approaches.

\begin{tabular}{lccc}
\hline Method & $\begin{array}{c}\text { Displacement } \\
(\mu \mathrm{m})\end{array}$ & Frequency $(\mathrm{Hz})$ & Stress (MPa) \\
\hline TLBO-ANFIS & 12042.30 & 61.44 & 314.70 \\
Jaya-ANFIS & 12581.11 & 67.76 & 333.68 \\
$\mathrm{X}_{\text {optimal }}=\left[t=0.4 \mathrm{~mm}, d_{1}=6.96 \mathrm{~mm}, d_{3}=6.91 \mathrm{~mm}, d_{4}=\right.$ \\
\hline
\end{tabular}

\section{Sensitivity Analysis}

The sensitivity analysis was an important step to identify and control the significant design variables. Based on the experimental data from Table 3, the sensitivity of each controllable parameters on each response was analyzed via using a statistical analysis, as depicted in Figure 13.

The contribution of thickness, $t$, on the displacement and stress were almost similar while the frequency was changed sharply with a small change of this parameter. Also, the results indicated that the displacement and stress are reduced when the thickness is increased. Meanwhile, the frequency was enhanced with an increase of thickness.

The length, $d_{1}$, was increased from $5 \mathrm{~mm}$ to $7.5 \mathrm{~mm}$, the displacement was also improved, and this change was 
TABLE 12: Comparison between the optimal, FEA, and experimental results.

\begin{tabular}{lccccc}
\hline Performances & Optimal results & FEA & Experiment & $\begin{array}{c}\text { Errors between } \\
\text { optimized and FEA } \\
\text { results }\end{array}$ & $\begin{array}{c}\text { Errors between } \\
\text { optimized and } \\
\text { experimental results }\end{array}$ \\
\hline Displacement $(\mu \mathrm{m})$ & 12581.11 & 12052.70 & 12014.96 & $4.38 \%$ & $4.71 \%$ \\
Frequency $(\mathrm{Hz})$ & 67.76 & 64.37 & 63.97 & $5.26 \%$ & $5.93 \%$ \\
Stress $(\mathrm{MPa})$ & 333.68 & 321.67 & 320.33 & $3.73 \%$ & $4.16 \%$ \\
\hline
\end{tabular}

quickly. In contrast, the stress was reduced quickly in this range. Meanwhile, in the range from $7.5 \mathrm{~mm}$ to $10 \mathrm{~mm}$, the displacement was decreased sharply but stress was lowered slowly. Besides, it showed that the frequency is not a little bit sensitive to $d_{1}$.

The displacement was not much sensitivity to the length of $d_{3}$. With $d_{1}$ in the range from $5 \mathrm{~mm}$ to $7.5 \mathrm{~mm}$, the frequency was decreased but the stress was increased sharply. In the range from $7.5 \mathrm{~mm}$ to $10 \mathrm{~mm}$, the frequency was raised slowly but the stress was decreased quickly.

With $d_{4}$ in the range $15 \mathrm{~mm}$ to $20 \mathrm{~mm}$, the displacement was so sensitive to this parameter because it was linearly decreased but the frequency was increased. In the range from $15 \mathrm{~mm}$ to $17.5 \mathrm{~mm}$, the stress was a little bit raised but, in the range from $17.5 \mathrm{~mm}$ to $20 \mathrm{~mm}$, the stress was reduced.

\section{Validations}

To validate the robustness and accuracy of the optimal performances of the proposed DCJ, the FEA and experiments were conducted. Using the optimal design variables, corresponding to $t=0.4 \mathrm{~mm}, d_{1}=6.96 \mathrm{~mm}, d_{3}=6.91 \mathrm{~mm}, d_{4}$ $=15.64 \mathrm{~mm}$, the prototype was fabricated by wire electrical discharged machining.

As seen in Table 12, the deviation errors between the optimal results with FEA and experimental ones were within from $3.73 \%$ to $5.93 \%$. These errors were relatively small. However, there were also some sources such as meshing, manufacturing, and material errors that could decrease those errors. To sum up, the proposed hybrid optimization algorithm was robust and effective in optimizing the DCJ.

Based on the soft computing approach and intelligent technique, the results of this paper would help to transfer from academic study to industry in the future. It was evidenced that the proposed method is a robust and effective tool.

\section{Comparison of Compliant Joints}

The DCJ was compared with traditional compliant joints via FEA. As seen in Figure 14, the common conventional compliant joints are, for example, leaf joint (LJ) [10], small pivot joint (SPJ) [11], right circular joint (RCJ) [12], and hyperbolic joint (HJ) [13]. The material Al 7075 was chosen in comparison. And then, the strain energy of each joint was computed. According to the Castigliano's theorem [20], the strain energy $U$ of a joint is calculated as follows:

$$
U=\frac{F}{2} \delta_{y}=\frac{F^{2}}{2 k}=\frac{F^{2} l^{3}}{6 E I}
$$

where $k$ is the stiffness of the joint.

A force from $2 \mathrm{~N}$ to $10 \mathrm{~N}$ was used. The boundary conditions of each joint were the same. Compared with previous joints, the results revealed that the displacement and strain energy of DCJ are better than those of other joints, as seen in Figures 15 and 16.

\section{Conclusions}

This paper presented the optimal design of the DCJ for camera positioning system used in nanoindentation tester. A new hybrid approach of Taguchi method, the ANFIS, and the Jaya algorithm was developed. First, the data was collected using the Taguchi method. The MFs for the structure ANFIS were determined well through the Taguchi method. Then, the $S / N$ ratios were computed and the WF of each cost function was determined by established equations. Subsequently, ANFIS was developed to map the design variables and the performances. Finally, Jaya algorithm was utilized to optimize the multiple objectives, simultaneously.

The results indicated that the optimal parameters are found at $t=0.4 \mathrm{~mm}, d_{1}=6.96 \mathrm{~mm}, d_{3}=6.91 \mathrm{~mm}$, and $d_{4}=15.64 \mathrm{~mm}$. Also, the optimal displacement, frequency, and stress were about $12581.11 \mu \mathrm{m}, 67.76 \mathrm{~Hz}$, and $333.68 \mathrm{MPa}$, respectively. These values were satisfied with the requirements. The validation results were in a good agreement with the predicted ones. The proposed hybrid optimization algorithm was robust and effective procedure for the DCJ. The proposed DCJ was also a potential candidate for industrial applications as compared with traditional compliant joints.

\section{Data Availability}

The data used to support the findings of this study are included within the article.

\section{Conflicts of Interest}

The authors declare that there are no conflicts of interest regarding the publication of this article. 


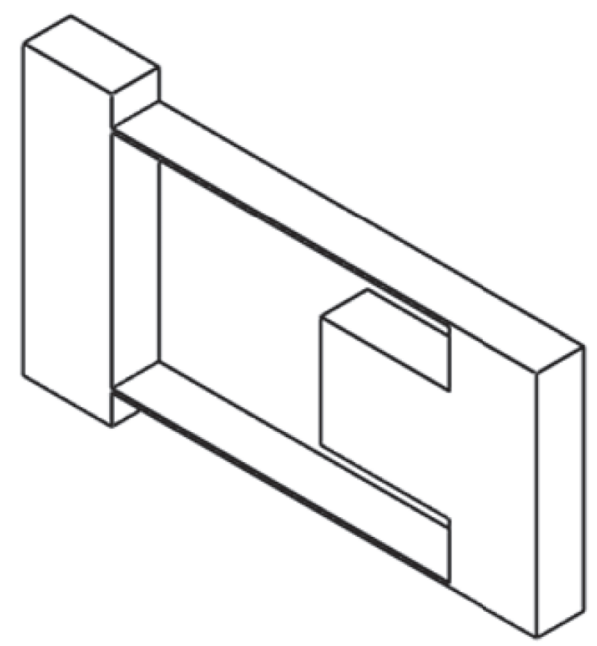

(a)



(c)

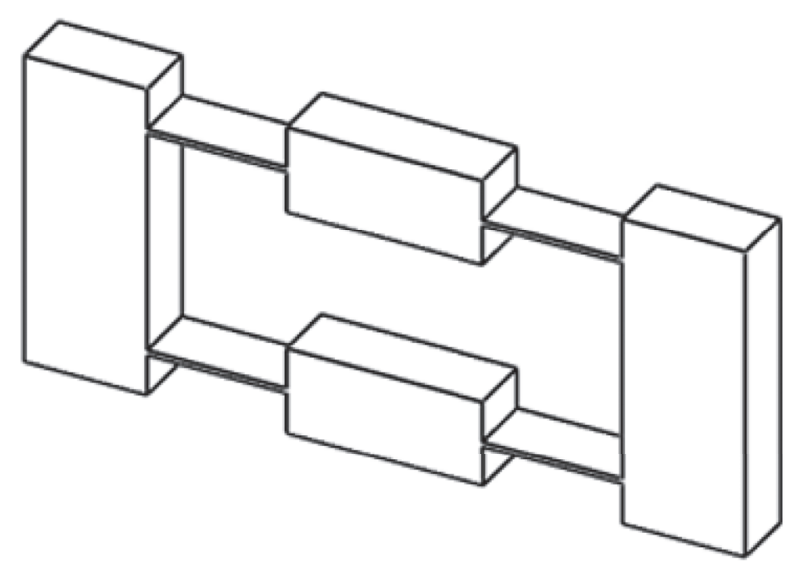

(b)

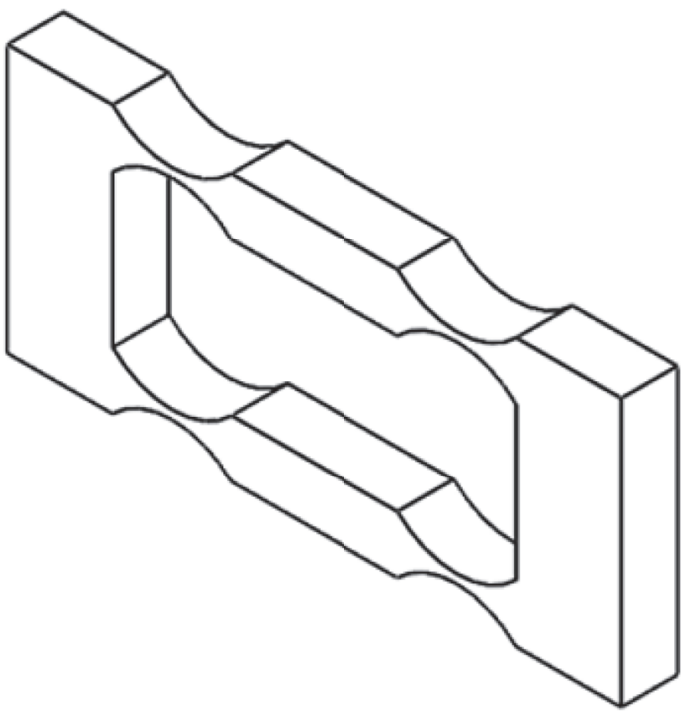

(d)

FIgURE 14: Common compliant joints: (a) LJ, (b) SPJ, (c) RCJ, and (d) HJ.

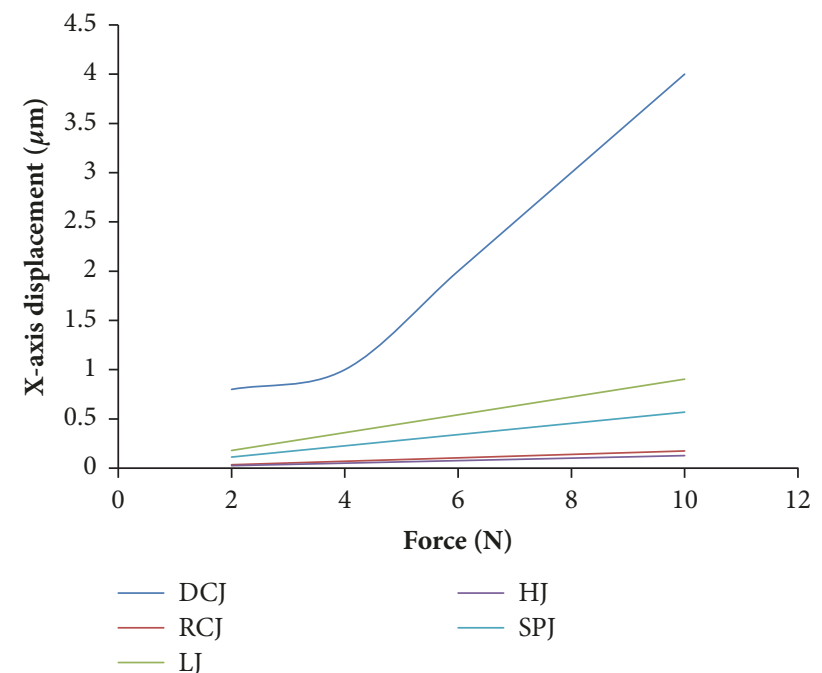

FIGURE 15: Comparison for $\mathrm{x}$-axis displacement of different joints (units in $\mu \mathrm{m}$ ).

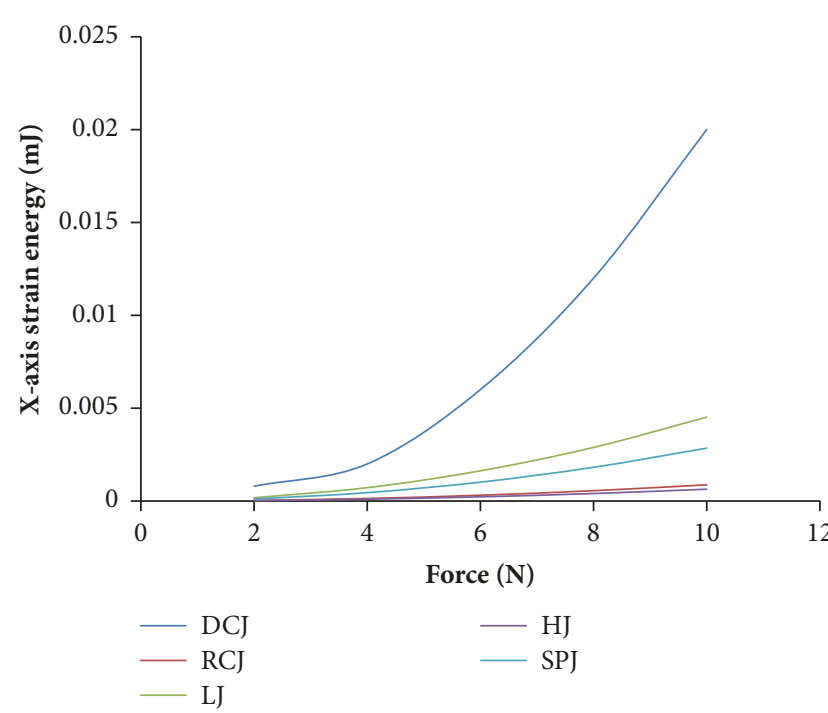

FIGURE 16: X-axis strain energy of different joints (units in $\mathrm{mJ}$ ). 


\section{Acknowledgments}

This research is funded by Vietnam National Foundation for Science and Technology Development (NAFOSTED) under Grant no. 107.01-2016.20.

\section{References}

[1] R. F. Fung, Y. L. Hsu, and M. S. Huang, "System identification of a dual-stage XY precision positioning table," Precision Engineering, vol. 33, no. 1, pp. 71-80, 2009.

[2] S. Polit and J. Dong, "Development of a high-bandwidth $\mathrm{XY}$ nanopositioning stage for high-rate micro-/nanomanufacturing," IEEE/ASME Transactions on Mechatronics, vol. 16, no. 4, pp. 724-733, 2011.

[3] Y. K. Yong and T.-F. Lu, "The effect of the accuracies of flexure hinge equations on the output compliances of planar micromotion stages," Mechanism and Machine Theory, vol. 43, no. 3, pp. 347-363, 2008.

[4] https://www.anton-paar.com.

[5] W. O'Brien, "Long-range motion with nanometer precision," Photonics Spectra, vol. 39, no. 6, pp. 80-81, 2005.

[6] M. Gauthier and E. Piat, "Control of a particular micro-macro positioning system applied to cell micromanipulation " IEEE Transactions on Automation Science and Engineering, vol. 3, no. 3, pp. 264-271, 2006.

[7] G. Dai, F. Pohlenz, H.-U. Danzebrink, M. Xu, K. Hasche, and G. Wilkening, "Metrological large range scanning probe microscope," Review of Scientific Instruments, vol. 75, no. 4, pp. 962-969, 2004.

[8] T. Hausotte, G. Jaeger, E. Manske, N. Hofmann, and N. Dorozhovets, "Application of a positioning and measuring machine for metrological long-range scanning force microscopy," in Optics \& Photonics, vol. 5878 of Proceedings of SPIE, p. 587802, San Diego, Calif, USA, 2005.

[9] M. J. Chung, Y. H. Yee, and D. H. Cha, "Development of auto focus actuator for camera phone by applying piezoelectric single crystal," in International Symposium on Optomechatronic Technologies, Proceedings of SPIE, p. 671507, International Society for Optics and Photonics, Lausanne, Switzerland, 2007.

[10] W. Y. Hsu, C. S. Lee, P. J. Chen et al., "Development of the fast astigmatic auto-focus microscope system," Measurement Science and Technology, vol. 20, no. 4, Article ID 045902, 2009.

[11] K.-C. Fan, C.-L. Chu, and J.-I. Mou, "Development of a lowcost autofocusing probe for profile measurement," Measurement Science and Technology, vol. 12, no. 12, pp. 2137-2146, 2001.

[12] C. Kim, M.-G. Song, Y. Kim et al., "Design of an auto-focusing actuator with a flexure-based compliant mechanism for mobile imaging devices," Microsystem Technologies, vol. 19, no. 9-10, pp. 1633-1644, 2013.

[13] M.-G. Song, H.-W. Baek, N.-C. Park et al., "Development of small sized actuator with compliant mechanism for optical image stabilization," IEEE Transactions on Magnetics, vol. 46, no. 6, pp. 2369-2372, 2010.

[14] T. T. Nguyen, H. G. Le, T. Dao, and S. Huang, "Evaluation of structural behaviour of a novel compliant prosthetic anklefoot," in Proceedings of the International Conference on Mechanical, System and Control Engineering (ICMSC '17), pp. 58-62, St. Petersburg, Russia, May 2017.

[15] M. Noh, S.-W. Kim, S. An, J.-S. Koh, and K.-J. Cho, "Fleainspired catapult mechanism for miniature jumping robots," IEEE Transactions on Robotics, vol. 28, no. 5, pp. 1007-1018, 2012.
[16] B. Edin, L. Beccai, L. Ascari, S. Roccella, J. Cabibihan, and M. Carrozza, "Bio-inspired approach for the design and characterization of a tactile sensory system for a cybernetic prosthetic hand," in Proceedings of the IEEE International Conference on Robotics and Automation (ICRA '06), pp. 1354-1358, Orlando, Fla, USA, 2006.

[17] U. G. K. Wegst, H. Bai, E. Saiz, A. P. Tomsia, and R. O. Ritchie, "Bioinspired structural materials," Nature Materials, vol. 14, no. 1, pp. 23-36, 2015.

[18] A. Ananthanarayanan, A. Mojtaba, and S. Kim, "Towards a bioinspired leg design for high-speed running," Bioinspiration \& Biomimetics, vol. 7, no. 4, Article ID 046005, 2012.

[19] A. T. Asbeck and M. R. Cutkosky, "Designing Compliant Spine Mechanisms for Climbing," Journal of Mechanisms and Robotics, vol. 4, no. 3, p. 031007, 2012.

[20] C. E. Doyle, J. J. Bird, T. A. Isom et al., "Avian-inspired passive perching mechanism for robotic rotorcraft," in Proceedings of the IEEE/RSJ International Conference on Intelligent Robots and Systems (IROS '11), pp. 4975-4980, San Francisco, Calif, USA, September 2011.

[21] C. E. Doyle, J. J. Bird, T. A. Isom et al., "An Avian-Inspired Passive Mechanism for Quadrotor Perching," IEEE/ASME Transactions on Mechatronics, vol. 18, no. 2, pp. 506-517, 2013.

[22] B. B. Edin, L. Ascari, L. Beccai, S. Roccella, J.-J. Cabibihan, and M. C. Carrozza, "Bio-inspired sensorization of a biomechatronic robot hand for the grasp-and-lift task," Brain Research Bulletin, vol. 75, no. 6, pp. 785-795, 2008.

[23] A. C. Etoundi, R. Vaidyanathan, and S. C. Burgess, "A bioinspired condylar hinge joint for mobile robots," in Proceedings of the 2011 IEEE/RSJ International Conference on Intelligent Robots and Systems (IROS 2011), pp. 4042-4047, San Francisco, CA, September 2011.

[24] A. M. Hoover, S. Burden, X.-Y. Fu, S. Shankar Sastry, and R. S. Fearing, "Bio-inspired design and dynamic maneuverability of a minimally actuated six-legged robot," in Proceedings of the EMBS International Conference on Biomedical Robotics and Biomechatronics (BioRob 2010), pp. 869-876, Tokyo, Japan, September 2010.

[25] G. Kim and J. Kim, "Compliant bistable mechanism for low frequency vibration energy harvester inspired by auditory hair bundle structures," Smart Materials and Structures, vol. 22, no. 1, p. 014005, 2013.

[26] H. Kozuka, J. Arata, K. Okuda et al., "A bio-inspired compliant parallel mechanism for high-precision robots," in Proceedings of the 2012 IEEE International Conference on Robotics and Automation (ICRA), pp. 3122-3127, St Paul, MN, USA, May 2012.

[27] G. Lau, H. Lim, J. Teo, and Y. Chin, "Lightweight mechanical amplifiers for rolled dielectric elastomer actuators and their integration with bio-inspired wing flappers," Smart Materials and Structures, vol. 23, no. 2, p. 025021, 2014.

[28] Y.-L. Park, B.-R. Chen, N. O. Pérez-Arancibia et al., "Design and control of a bio-inspired soft wearable robotic device for anklefoot rehabilitation," Bioinspiration \& Biomimetics, vol. 9, no. 1, Article ID 016007, 2014.

[29] B. F. Seitz, B. Goldberg, N. Doshi et al., "Bio-inspired mechanisms for inclined locomotion in a legged insect-scale robot," in Proceedings of the 2014 IEEE International Conference on Robotics and Biomimetics (ROBIO), pp. 791-796, Bali, Indonesia, December 2014.

[30] T.-P. Dao and S.-C. Huang, "Compliant thin-walled joint based on zygoptera nonlinear geometry," Journal of Mechanical Science and Technology, vol. 31, no. 3, pp. 1293-1303, 2017. 
[31] N. Le Chau, V. A. Dang, H. G. Le, and T.-P. Dao, "Robust Parameter Design and Analysis of a Leaf Compliant Joint for Micropositioning Systems," Arabian Journal for Science and Engineering, vol. 42, no. 11, pp. 4811-4823, 2017.

[32] S.-C. Huang and T.-P. Dao, "Design and computational optimization of a flexure-based XY positioning platform using FEAbased response surface methodology," International Journal of Precision Engineering and Manufacturing, vol.17, no. 8, pp.10351048, 2016.

[33] S.-C. Huang and T.-P. Dao, "Multi-objective optimal design of a 2-DOF flexure-based mechanism using hybrid approach of Grey-Taguchi coupled response surface methodology and entropy measurement," Arabian Journal for Science and Engineering, vol. 41, no. 12, pp. 5215-5231, 2016.

[34] T.-P. Dao and S.-C. Huang, "Design and multi-objective optimization for a broad self-amplified 2-DOF monolithic mechanism," Sädhanā, vol. 42, no. 9, pp. 1527-1542, 2017.

[35] T.-P. Dao, "Multiresponse optimization of a compliant guiding mechanism using hybrid Taguchi-grey based fuzzy logic approach," Mathematical Problems in Engineering, vol. 2016, Article ID 5386893, 17 pages, 2016.

[36] J. S. R. Jang, "ANFIS: adaptive-network-based fuzzy inference system," IEEE Transactions on Systems, Man, and Cybernetics, vol. 23, no. 3, pp. 665-685, 1993.

[37] R. V. Rao, K. More, J. Taler, and P. Ocłoń, "Dimensional optimization of a micro-channel heat sink using Jaya algorithm," Applied Thermal Engineering, vol. 103, pp. 572-582, 2016. 


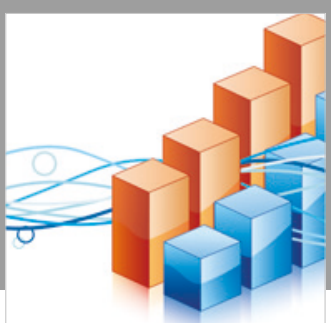

Advances in

Operations Research

\section{-n-m}

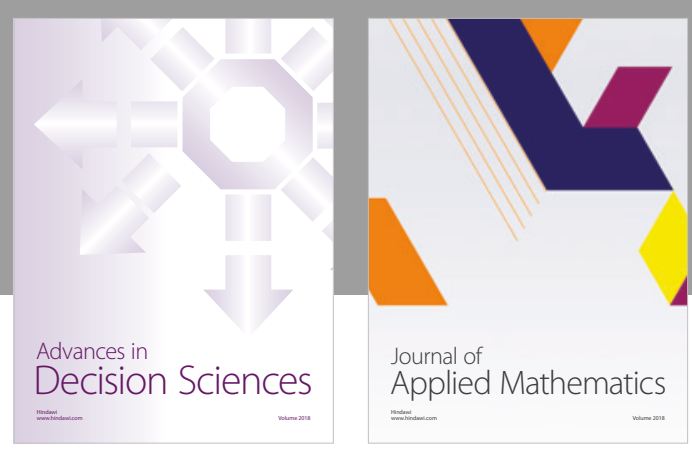

Journal of

Applied Mathematics
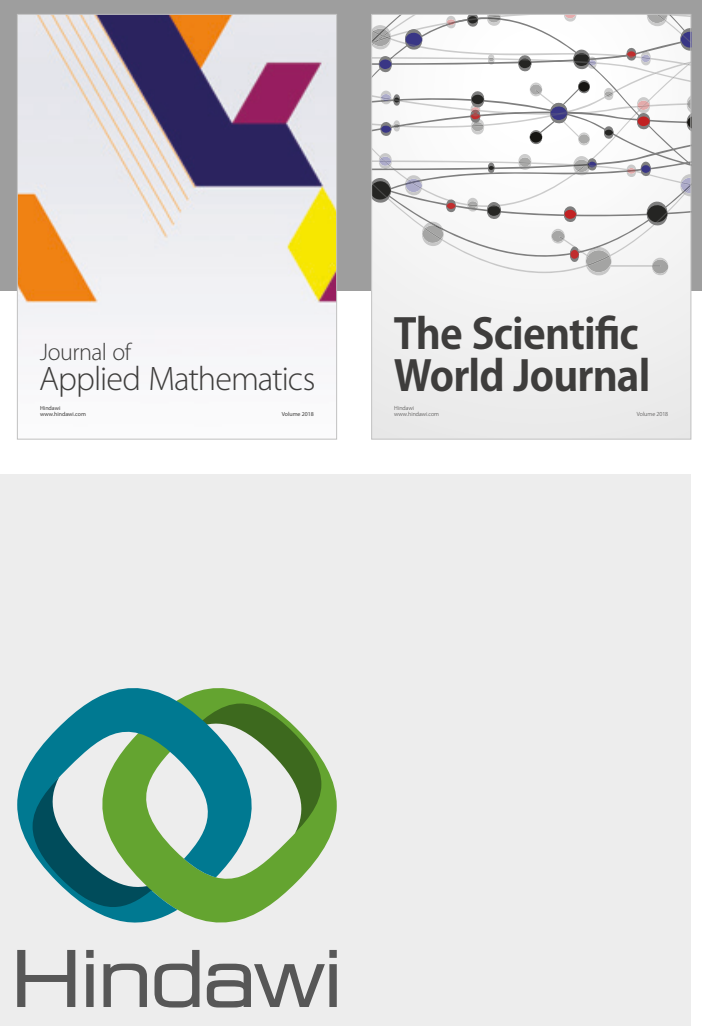

Submit your manuscripts at

www.hindawi.com

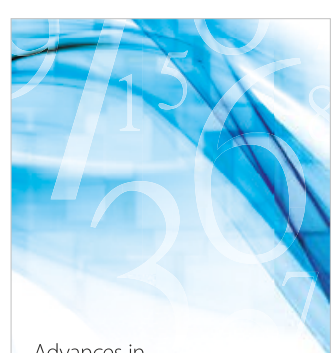

Advances in
Numerical Analysis
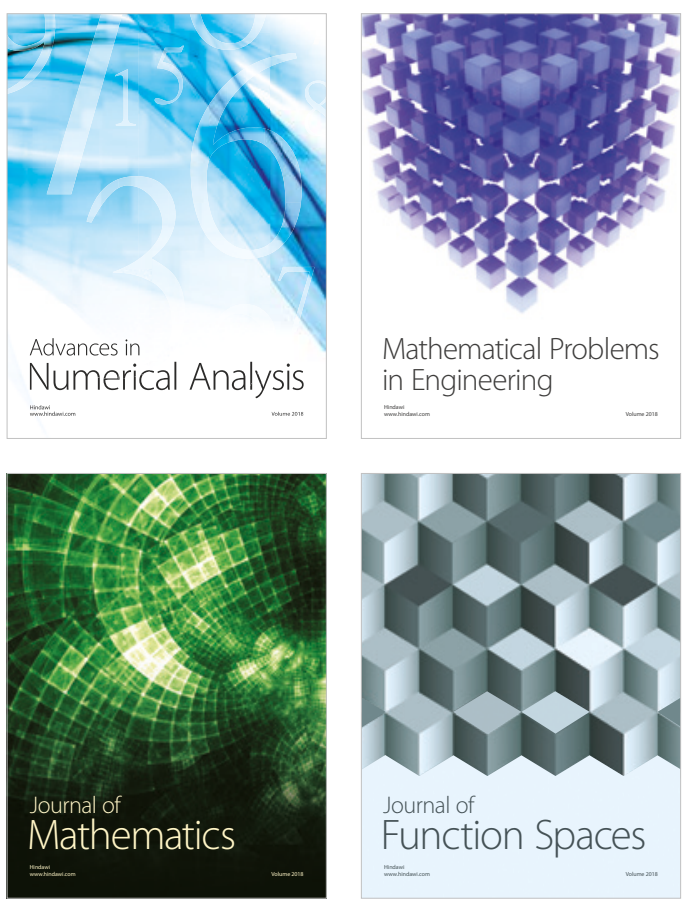

Mathematical Problems in Engineering



International Journal of

Differential Equations

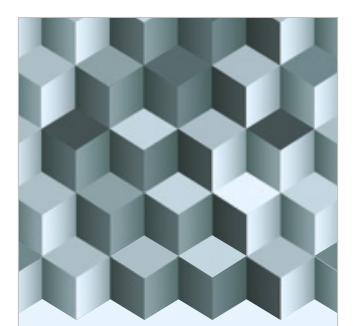

Journal of

Function Spaces



The Scientific

World Journal

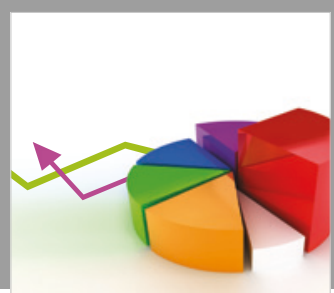

Journal of

Probability and Statistics
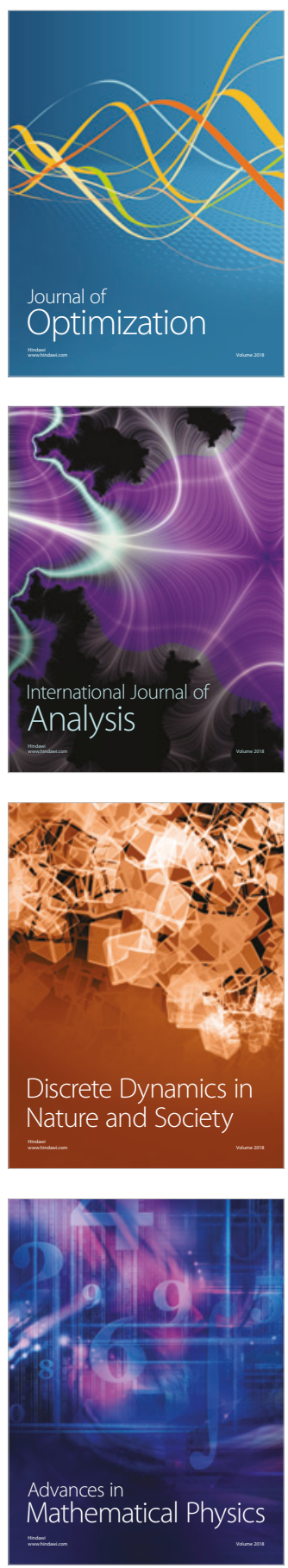